\title{
Colonoscopy with mucosal biopsies in young rats: A model for experimental gastroenterology
}

\author{
MAGDY EL-SALHY ${ }^{1,2}$, INGVILD HAUKAAS WENDELBO ${ }^{1,2}$, \\ DORIS GUNDERSEN ${ }^{3}$, JAN GUNNAR HATLEBAKK ${ }^{2}$ and TRYGVE HAUSKEN ${ }^{2}$ \\ ${ }^{1}$ Section for Gastroenterology, Department of Medicine, Stord Helse-Fonna Hospital, Stord; ${ }^{2}$ Section for Gastroenterology, \\ Institute of Medicine, University of Bergen, Bergen; ${ }^{3}$ Department of Research, Helse-Fonna, Haugesund, Norway
}

Received December 14, 2012; Accepted February 25, 2013

DOI: $10.3892 / \mathrm{mmr} .2013 .1422$

\begin{abstract}
Endoscopy is an important tool in the diagnosis, follow-up and management of several gastrointestinal diseases. In experimental studies, the course and progression of gastrointestinal lesions are followed by sacrificing animals at specific time intervals and the disease is usually assessed postmortem. This approach has several disadvantages, including the requirement of a large number of experimental animals and the inability to evaluate and follow the progression of the same lesion. The present study tested the feasibility of performing a total colonoscopy with mucosal biopsies in small, young rats. Eighteen adult male Wistar rats with an average bodyweight of $194 \mathrm{~g}$ (range, 166-232 g) were used. Three bowel preparation regimes with different fasting times and doses of sodiumpicosulfate and magnesium citrate were examined. A video gastroscope with a $4.9 \mathrm{~mm}$ outer diameter, $210 / 120^{\circ}$ up/down tip deflection, $103 \mathrm{~cm}$ working length, $140^{\circ}$ view field and a $2 \mathrm{~mm}$ working channel (GIF-N180, Olympus, Tokyo, Japan) was used. The rats were anesthetized by inhalation of isoflurane prior to and during colonoscopy. Biopsies were obtained using biopsy forceps through the endoscope working channel. The animals were allowed to recover and were monitored for $\sim 1 \mathrm{~h}$, following the procedure. Sections from the biopsy samples taken during colonoscopy and colon tissue obtained during postmortem laparotomy were stained with hematoxylin and eosin. The optimum results were obtained with the bowel preparation that involved rats fasting for $24 \mathrm{~h}$ and the administration of 1 and $2 \mathrm{ml}$ of sodium-picosulfate/magnesium citrate at 24 and $12 \mathrm{~h}$ prior to colonoscopy, respectively. Colonoscopy was performed with minor complications, with the only difficulty arising when maneuvering the endoscope past the hepatic flexure. This was achieved by manual rotation of the endoscope shift to the right and left of a two-way endoscope.
\end{abstract}

Correspondence to: Professor Magdy El-Salhy, Section for Gastroenterology, Department of Medicine, Stord Helse-Fonna Hospital, Tysevegen 64, Box 4000, 5409 Stord, Norway

E-mail: magdy.el-salhy@helse-fonna.no

Key words: biopsy, colonoscopy, rats, mucosa, perforation
Taking biopsies with the forceps at full jaw length and swing resulted in perforation, which may be avoided by obtaining biopsies with the outer tip of the biopsy forceps.

\section{Introduction}

Endoscopy is an important tool in the diagnosis, follow-up and management of several gastrointestinal diseases, including inflammatory bowel disease and cancer. The endoscopic features and results of biopsy examinations are the parameters used for evaluation in clinical practice. In experimental studies, the course and progression of gastrointestinal lesions are followed by sacrificing animals at specific time intervals. The disease is usually assessed postmortem. This approach has several disadvantages: i) it requires a large number of experimental animals; ii) it is not possible to follow the progression and evaluation of the same lesion and iii) the findings are difficult to compare with biopsy samples obtained from patients by endoscopy.

Several studies have reported on attempts to perform colonoscopy in rats (1-9). However, in these studies, the rats were either large and old, the bowel was not optimally prepared, a total colonoscopy was not performed or no biopsies were taken. The present study tested the feasibility of performing a total colonoscopy with mucosal biopsies in small, young rats.

\section{Materials and methods}

Rats. Eighteen adult male Wistar rats (Hannover GALAS, Taconic Farms, Laven, Denmark) with a mean average bodyweight of $194 \mathrm{~g}$ (range, 166-232 $\mathrm{g}$ ) and an average age of 6-7 weeks were used. The rats were housed singly in Macrolon III cages and fed a standard diet ad libitum (B\&K Universal AS, Nittedal, Norway), consisting of cereal products $(88.5 \%)$, soy protein $(6 \%)$, animal protein $(2.5 \%)$, soy oil $(0.5 \%)$, and vitamin, mineral and amino acid supplements $(2.5 \%)$. Water was also provided ad libitum. The rats were maintained at a temperature of $21 \pm 1^{\circ} \mathrm{C}$, a relative humidity of $55 \pm 5 \%$ and under a 12-h light/dark cycle. The animals were left in cages for a minimum of 7 days in order to acclimatize prior to colonoscopy. While the rats were fasting, a grid floor was used so that the feces would fall between the grid bars, thus preventing the rats from eating them. 
Bowel preparation. Three regimens for bowel cleansing were examined: i) the rats had free access to water, however, food was withdrawn $36 \mathrm{~h}$ prior to colonoscopy. The rats received a gastric dose of $1 \mathrm{ml}$ of Picoprep ${ }^{\circledR}$ (Ferring, Saint-Prex, Switzerland) followed by $2 \mathrm{ml}$ of water, $24 \mathrm{~h}$ prior to colonoscopy. Picoprep is prepared in $150 \mathrm{ml}$ of water and contains $10 \mathrm{mg}$ of sodium sulfate, $3.5 \mathrm{~g}$ of magnesium oxide and $12 \mathrm{~g}$ of citric acid. Picoprep was introduced via an $8.5 \mathrm{~cm}$ long, $2.5 \mathrm{~mm}$ round-tip Teflon feeding gauge (AgnTho's AB, Lidingö, Sweden); ii) the rats were fasted for $24 \mathrm{~h}$ and then received a gastric dose of $1 \mathrm{ml}$ of Picoprep followed by $2 \mathrm{ml}$ of water, $24 \mathrm{~h}$ prior to colonoscopy and iii) the rats were fasted for $24 \mathrm{~h}$ and received gastric doses of 1 and $2 \mathrm{ml}$ of Picoprep at 24 and $12 \mathrm{~h}$ prior to colonoscopy, respectively. Following this, $2 \mathrm{ml}$ of water was provided to the rats in both groups.

Colonoscopy and biopsy. The rats were anesthetized by inhalation of isoflurane (Merk Pharmaceutical co., Inc., West Point, PA, USA) prior to and during colonoscopy. They were then placed into a supine position and secured onto an acrylic surgical table (World Precision Instruments, Sarasota, FL, USA). The rats were placed on a warming pad (Gaymar T/ Pad, Gaymar Industries, Orchard Park, NY, USA) using a heat therapy pump (TP500 t/Pump, Gaymar Industries) to maintain normothermia during the procedure. A video gastroscope with a $4.9 \mathrm{~mm}$ outer diameter, $210 / 120^{\circ}$ up/down tip deflection, $103 \mathrm{~cm}$ working length, $140^{\circ}$ view field and a $2 \mathrm{~mm}$ working channel (GIF-N180, Olympus Medical System, Tokyo, Japan) was used. In addition to the gastroscope, a light source (CLV-180, 230V S EVIS EXERA II Xenon, Olympus Medical System), Olympus CV-180 possessor (EVIS EXERA II video center, Olympus Medical System) and a monitor (21 inch, Advan, Fremont, CA, USA) were used (Fig. 1). Biopsy samples were obtained by disposable biopsy forceps (EndoJaw FB-231K, Olympus Medical System) with a working length of $1550 \mathrm{~mm}$ and a minimum channel diameter of $2 \mathrm{~mm}$. These forceps have a jaw length of $3 \mathrm{~mm}$ and a fenestrated jaw swing diameter of $1.9 \mathrm{~mm}$. The top of the endoscope was lubricated with $2 \%$ lidocaine (Xylocaine ${ }^{\circledR}$, Astra Zeneca Pharmaceutics, Wilmington, UK) and introduced gently into the anus.

Surveillance and necropsy. Following the procedure, the animals were allowed to recover from anesthesia and were monitored for $\sim 1 \mathrm{~h}$. They were then sacrificed by inhalation of $\mathrm{CO}_{2}$ and a postmortem laparotomy was performed. The colon was dissected and its length from the anus to the cecum was measured. Tissue samples from the colon were collected for histological examination.

Histological examination. The biopsy samples obtained during colonoscopy and from the colon tissue during postmortem laparotomy were fixed in $4 \%$ buffered paraformaldehyde overnight, embedded in paraffin and then cut into $5-\mu \mathrm{m}$ sections. The sections were stained with hematoxylin and eosin. The depth of the colonic wall was measured in samples taken postmortem from rats by computer image analysis using Cell ${ }^{\wedge} \mathrm{D}$ software (Olympus Medical System).

The study was carried out in accordance with the Directive for the Protection of Vertebrate Animals used for Experimental and other Scientific Purposes of the European Union (86/609/

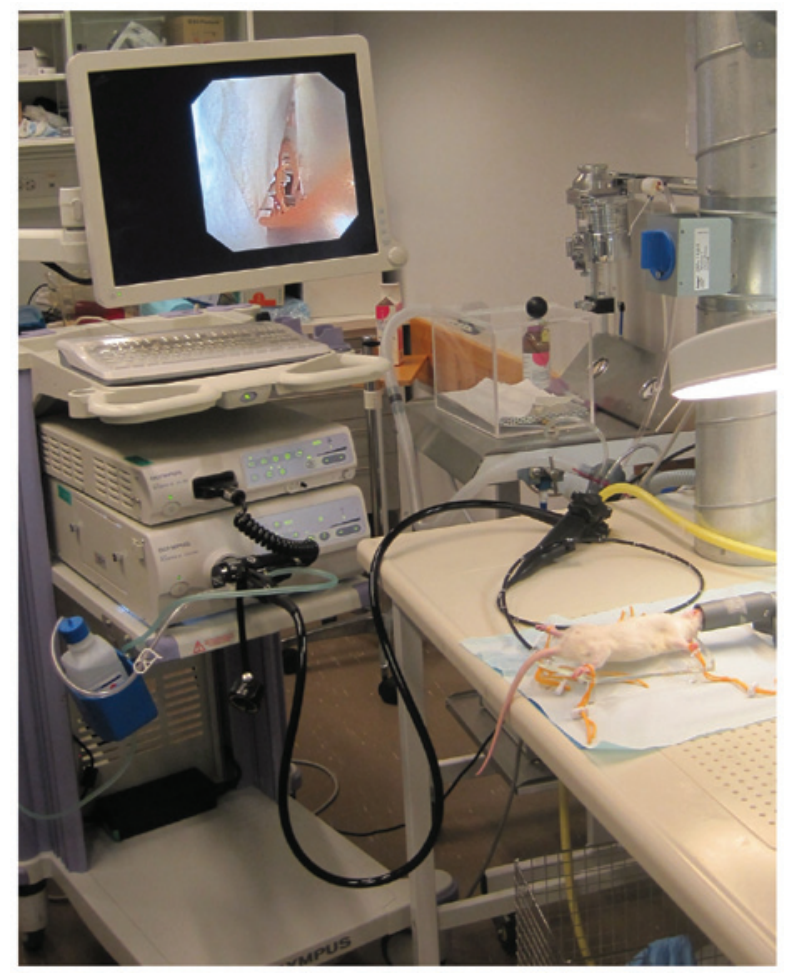

Figure 1. Laboratory and equipment used to perform colonoscopy in rats.

EEC), in compliance with the Helsinki Declaration. The local ethical committee for experimental animals approved the protocols of the study.

\section{Results}

Bowel preparation. The colon of rats that were fasted for $36 \mathrm{~h}$ and had received $1 \mathrm{ml}$ of Picoprep, $24 \mathrm{~h}$ prior to colonoscopy, were filled with solid feces, therefore hindering the observation of the lining mucosa, even following flushing with water. The colon of rats that were fasted for $24 \mathrm{~h}$ and had received $1 \mathrm{ml}$ of Picoprep, $24 \mathrm{~h}$ prior to colonoscopy, were considerably cleaner, however, solid feces were occasionally encountered. The colon of rats that were fasted for $24 \mathrm{~h}$ and received 1 and $2 \mathrm{ml}$ Picoprep at 24 and $12 \mathrm{~h}$ prior to colonoscopy, respectively, were completely clean of feces.

Colonoscopy, biopsy and histological examination. To facilitate the insertion of the endoscope and relaxation of the anal sphincter, a $2.5-\mathrm{mm}$ round-tip Teflon tube was inserted into the anus prior to insertion of the endoscope. The colonoscopy was straightforward as this is a relatively simple procedure for a physician with training in flexible endoscopy (Fig. 2). Rats have no splenic flexure and therefore the only difficulty arose when maneuvering the endoscope past the hepatic flexure (Fig. 3). When using a two-way endoscope (up and down), this can only be achieved by manual rotation of the endoscope shift to the right and left of a two-way endoscope. Abdominal manipulation increases the risk of perforation. The cecum was identified by the sudden increase in bowel diameter, transillumination of the abdominal wall and the presence of a small amount of solid feces in an otherwise clean colon. Inflation with air should be avoided, however, if necessary, this should 


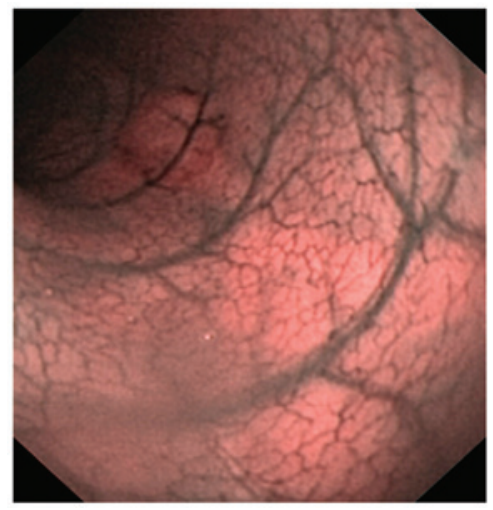

Figure 2. Appearance of the colonic mucosa on narrow-band imaging, which provides twice the viewable distance and offers a greater contrast between the blood vessels and the mucosa.

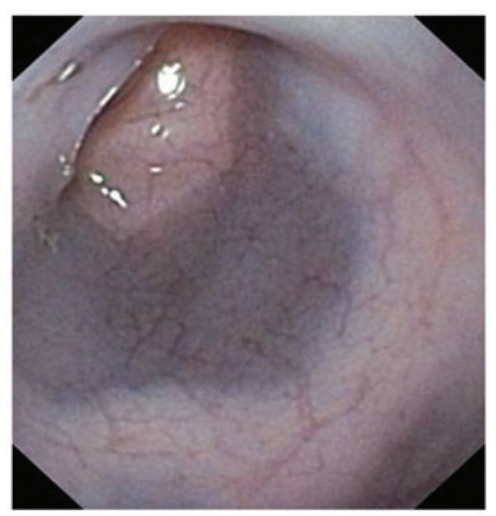

Figure 3. Sharp hepatic flexure of the rat colon.

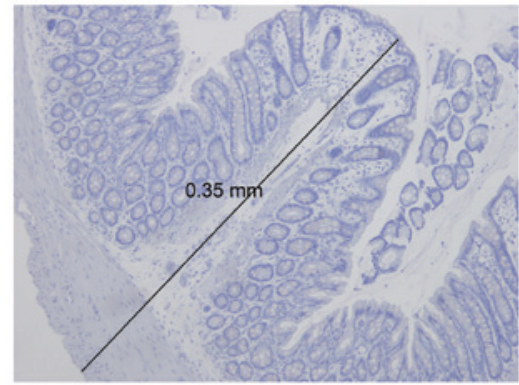

Figure 4. Measurement of the thickness of the colonic wall in a perpendicularly cut section.

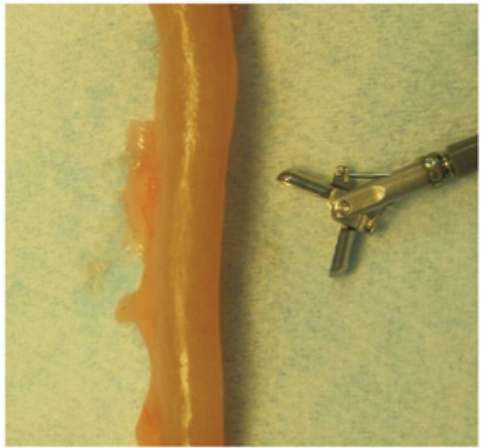

Figure 5. Comparison between the diameter of the rat colon and the jaws of the biopsy forceps used.

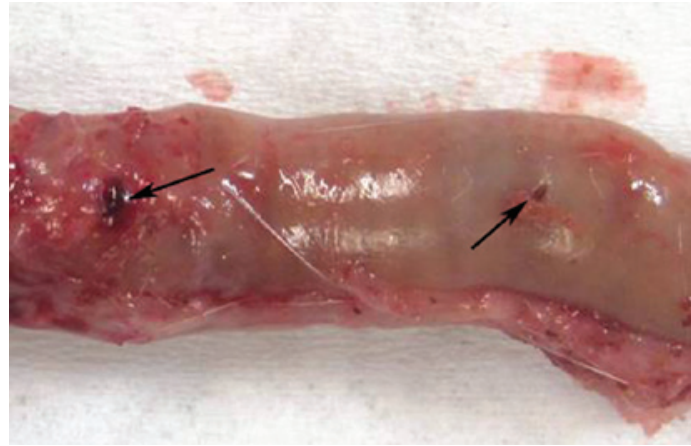

Figure 6. Macroscopic examination of the colon showing perforation (arrows) when biopsies were collected with the full length of the biopsy forceps.

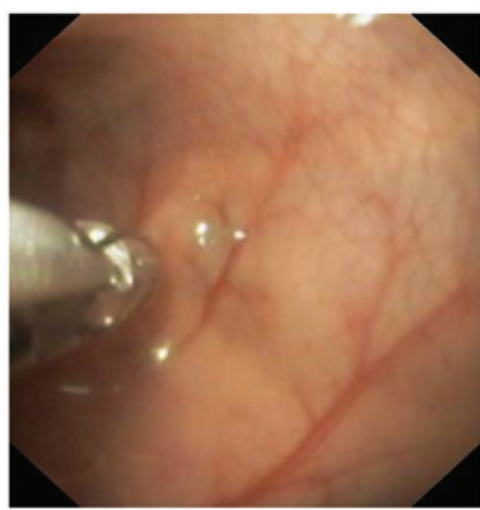

Figure 7. Endoscopic image showing the safest method to collect biopsies using the outer tip of the biopsy forceps.

A

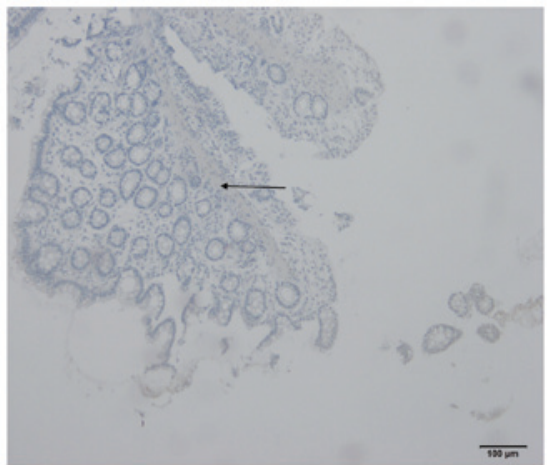

B

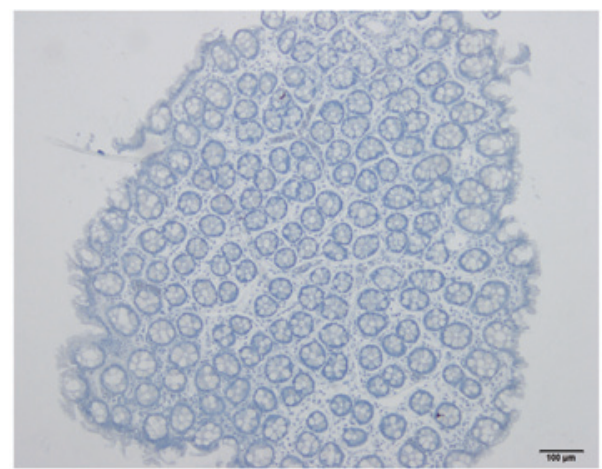

Figure 8. Photomicrographs of a biopsy obtained with the full length of the biopsy forceps containing epithelium, lamina propria and muscularis mucosa (arrow). (A) Biopsy taken with the tip of the biopsy forceps, which includes only the epithelium and lamina propria. (B) Hematoxylin and eosin stain. 
be performed using air under low pressure. The lumen was visualized by flushing with water to avoid abdominal distension. The time required to perform a total colonoscopy and to obtain three colonic biopsies varied between 6 and $10 \mathrm{~min}$.

The maximum thickness of the colon wall was $0.35 \mathrm{~mm}$ (Fig. 4) and the dimensions of the biopsy forceps were almost as large as the circumference of the rat colon (Fig. 5). Obtaining biopsies with the forceps full jaw length and swing resulted in perforation (Fig. 6). Perforation was avoided by obtaining the biopsies with the outer tip of the biopsy forceps (Fig. 7).

Histological examination of the biopsies collected from perforated rats revealed that they included the epithelium, lamina propria and muscularis mucosa (Fig. 8A). By contrast, those obtained with the tip of the biopsy forceps included only the epithelium and lamina propria (Fig. 8B), which is comparable with biopsies collected during a standard colonoscopy in humans.

Surveillance and necropsy. One rat died of aspiration caused by the gastric tube during bowel preparation and two rats died due to colonic perforation. A total colonoscopy was performed successfully in the remaining rats, which recovered well thereafter. Postmortem macroscopic examination of the abdomen and colon did not reveal any complications. The length of the colon from the anus to the cecum varied between 14 and $17 \mathrm{~cm}$.

\section{Discussion}

The results of the present study demonstrate that colonoscopy with mucosal biopsies is feasible in small, young rats. However, several aspects should be taken into consideration when using equipment designed for endoscopy in humans and when performed by an endoscopist only trained in conducting endoscopy in human patients.

Several studies examining colonoscopies were conducted in large and old rats weighing up to $780 \mathrm{~g}(2,3,5,8)$. Although this simplified the procedure, it has several disadvantages, including the introduction of age as a limiting factor in the experimental procedure and limitiation of long-term follow-up. In addition, only partial colonoscopies have been performed in rats of $200 \mathrm{~g}$ bodyweight $(1,4)$. In the present study, total colonoscopy was performed successfully in 6-7-week-old rats weighing <200 g.

Bowel preparation prior to colonoscopy is essential for a successful procedure. Bowel preparation varies between studies, from no bowel preparation at all $(2,3)$ to lavage with water through a gastric feeding tube (4), fasting for $24 \mathrm{~h}$ and flushing with water prior to colonoscopy (1) and fasting for $56 \mathrm{~h}$ and administration of oral phosphosoda once or twice in addition to a saline solution enema prior to colonoscopy $(5,8)$. The latter regime, which is the most rigorous, results in a clean bowel, however, often distresses the rats $(5,8)$. In total, $1.6 \%$ of rats subjected to this regime died during bowel preparation (8). Rats have a tendency to ingest their feces during starvation. To prevent this in the present study, the rats were housed on a grid floor in order that feces would fall through to the bottom of the cage and become inaccessible, however, following fasting for $36 \mathrm{~h}$, a number of rats were able to ingest their feces. Fasting for $24 \mathrm{~h}$ with 2 doses of Picoprep provided the most effective preparation.

The endoscope used to perform colonoscopy in rats is an important factor with regard to successful procedure, with three of its properties particularly relevant, i.e., its diameter, flexibility and tip movements in the up and down, and right to left direction. A small endoscope is simpler to insert and minimizes the risk of perforation, as would a flexible endoscope which is capable of more readily following the course of the colon. Movement of the endoscope tip facilitates its navigation through the sharp bend of the hepatic flexure. Rat colons have been examined in a number of studies using a rigid endoscope with a diameter of 2.2 or $2.7 \mathrm{~mm}(2,4)$, and with a flexible endoscope exhibiting tip mobility in four directions (with a diameter of 5.2-5.9 mm) $(1,5,8)$. A total colonoscopy was achieved when using a flexible endoscope with the ability to maneuvre the tip in four directions and with a diameter of $5.9 \mathrm{~mm}(5,8)$. In the present study, colonoscopy was performed using an endoscope with a diameter of $4.9 \mathrm{~mm}$, which is the smallest available with a working channel for collecting biopsy samples. The tip of the endoscope was only able to be maneuvered up and down. The diameter of the endoscope did not cause any complications, however, the endoscope had to be manually rotated and shifted right and left to enable it to pass the hepatic flexure.

While previous studies have reported the collection of biopsies during colonoscopy in rats $(2,3)$, several factors including the rate of perforation and other complications were not reported. Preliminary experiments with the same biopsy forceps used in the present study demonstrated that colonoscopy was feasible without a risk of perforation (1). The present findings demonstrate that the risk of perforation is high when collecting biopsy samples, however, the procedure can be successful if caution is exercised.

\section{Acknowledgements}

This study was supported by a grant from Helse-Fonna.

\section{References}

1. Vermeulen W, De Man JG, Nullens S, Pelckmans PA, De Winter BY and Moreels TG: The use of colonoscopy to follow the inflammatory time course of TNBS colitis in rats. Acta Gastroenterol Belg 74: 304-311, 2011.

2. Mann NS, Mann SK and Cheung EC: Fiberoptic colonoscopic study of experimental chemical colitis. Gastrointest Endosc 26: 28-40, 1980.

3. Mann NS and Demers LM: Experimental colitis studied by colonoscopy in the rat: effect of indomethacin. Gastrointest Endosc 29: 77-82, 1983.

4. Ahn BO, Ko KH, Oh TY, Cho H, Kim WB, Lee KJ, Cho SW and Hahm KB: Efficacy of use of colonoscopy in dextran sulfate sodium induced ulcerative colitis in rats: the evaluation of the effects of antioxidant by colonoscopy. Int J Colorectal Dis 16: 174-181, 2001.

5. Hamilton SR, Zhang SZ, O'Ceallaigh D and McAvinchey D: Growth characteristics of autochthonous experimental colonic tumors as assessed by serial colonoscopic measurement in rats. Gastroenterology 91: 1511-1520, 1986.

6. Hull CC, Stellato TA, Ament AA, Gordon N and Galloway P: Endoscopic and radiographic evaluation of the murine colon. Cancer 66: 2528-2532, 1990.

7. Karas JR, Essani R, Haughn C, Uchal M, Bishawi MM and Bergamaschi R: Colonoscopic injection for murine solid cecal cancer model. Surg Endosc 25: 2956-2959, 2011.

8. Haughn C, Uchal M, Raftopoulos Y, Rossi S, Santucci T, Torpey M, Pollice A, Yavuz Y, Marvik R and Bergamaschi R: Development of a total colonoscopy rat model with endoscopic submucosal injection of the cecal wall. Surg Endosc 20: 270-273, 2006.

9. Narisawa T, Wong CQ and Weisburger JH: Evaluation of endoscopic examination of colon tumors in rats. Am J Dig Dis 20: 928-934, 1975. 